\title{
Controle de Plantas daninhas com Herbicidas Cloroacetamidas em Sistemas Convencional E De Semeadura direta ${ }^{1}$
}

\author{
Weed Control with Chloroacetamide Herbicides in Conventional and No-Tillage Systems
}

FERRI, M.V.W. ${ }^{2}$ e VIDAL, R.A. ${ }^{3}$

\begin{abstract}
RESUMO - Os sistemas de manejo do solo podem alterar a bioatividade dos herbicidas cloroacetamidas e influir na sua persistência no solo, seletividade às culturas e eficácia de controle das plantas daninhas. Um experimento foi conduzido na Universidade Federal do Rio Grande do Sul, durante o ano agrícola de 2000/2001, para avaliar a eficácia de controle das plantas daninhas pelos herbicidas cloroacetamidas, em solo Argissolo Vermelho, manejado sob semeadura direta e preparo convencional. Foram avaliados os herbicidas acetochlor e alachlor, cada um na dose de $3.360 \mathrm{~g}$ ha, metolachlor a $2.400 \mathrm{~g}$ ha, s-metolachlor a $1.440 \mathrm{~g}$ ha, s-metolachlor + antídoto à 1.440 e $1.800 \mathrm{~g} \mathrm{ha}^{-1}$, respectivamente, além da testemunha sem herbicida, nos sistemas convencionais e semeadura direta. As avaliaçóes foram de eficácia de controle, população de plantas daninhas e matéria seca da parte aérea das plantas daninhas, realizadas aos 30 e 50 dias após aplicação dos tratamentos (DAT). Os herbicidas cloroacetamidas foram mais eficientes no controle das plantas daninhas no preparo convencional, comparado à semeadura direta, em ambas as épocas de avaliação. Os herbicidas acetochlor, alachlor a $3.600 \mathrm{~g}$ ha e s-metolachlor + protetor a $2.400 \mathrm{~g}$ ha foram mais eficientes no controle destas plantas, em relação aos demais herbicidas e formulações. A produção de matéria seca e a população das plantas daninhas foram menores no preparo convencional.
\end{abstract}

Palavras-chave: persistência, manejo de solos, metolachlor, acetochlor e alachlor.

\begin{abstract}
Soil management systems can affect chloroacetamide herbicide bioactivity and its soil persistence, crop selectivity and weed control. An experiment was carried out at the University of Rio Grande do Sul, Brazil, during the growing season 2000/2001, to evaluate the weed control efficacy of the chloroacetamide herbicides, in a Paleudult soil, under conventional tillage and notillage systems. The following treatments were tested: acetochlor and alachlor, each at the rate of $3.360 \mathrm{~g} \mathrm{ha}^{-1}$, metolachlor at $2.400 \mathrm{~g} \mathrm{ha}^{1}$, s-metolachlor at $1.440 \mathrm{~g} \mathrm{ha}^{-1}$, and s-metolachlor + protector at 1.440 and $1.800 \mathrm{~g} \mathrm{ha}$, respectively, besides a check plot without herbicide, applied under conventional and no-tillage systems. Assessments included weed population, weed dry matter and weed control at 30 and 50 days after herbicide application. The chloroacetamides herbicides were more effective in controlling weeds under conventional tillage, compared to no tillage, in both evaluation times. The herbicides acetochlor, alachlor at 3,600 $\mathrm{g} \mathrm{ha}^{1}$ and metolachlor + protector at 2,400 $\mathrm{g} \mathrm{ha}^{-1}$ were more efficient in controlling these plants when compared to the other herbicides and formulations. Dry biomass production and weed populations were lower under conventional tillage.
\end{abstract}

Key words: persistence, herbicides, soil management, metolachlor, acetochlor and alachlor.

\section{INTRODUÇÃO}

A expressão do potencial produtivo pelas culturas depende, entre outros fatores, da intensidade, da época e da duração do controle de plantas daninhas. O controle se faz necessário porque as plantas daninhas competem com as culturas por fatores imprescindíveis à expressão de seu potencial produtivo, como água, luz e nutrientes. As plantas daninhas também interferem nas culturas, por abrigarem insetos, patógenos de doenças e nematóides, além de

1 Recebido para publicação em 15.1.2002 e na forma revisada em 2.4.2003.

2 Eng.-Agr., Dr., Caixa Postal 138, 85550-000 Coronel Vivida-Pr, <mvwferri bol.com.br >. ${ }^{3}$ Eng.-Agr. Ph.D., professor do Dep. de Plantas de Lavoura da Faculdade de Agronomia da UFRGS, Av. Bento Gonçalves, 7712, 91501-970 Porto Alegre-RS. 
dificultarem ou inviabilizarem a sua colheita. A competição e/ou interferência originada pela presença de plantas daninhas nas lavouras normalmente resulta em prejuízos qualitativos e quantitativos à produção agrícola (Durigan et al., 1983; Pitelli \& Durigan, 1984; Fleck et al., 1989).

A ocorrência de plantas daninhas desde o início do ciclo de desenvolvimento das culturas pode acarretar perdas acentuadas de produtividade, principalmente se o controle destas plantas for realizado em época inadequada e/ ou for ineficiente. Entende-se por período total de prevenção da interferência entre plantas daninhas e culturas o período do ciclo no qual as culturas devem permanecer livres da interferência das plantas daninhas para melhor expressarem seu potencial produtivo. Em geral, em culturas anuais, como milho e soja, esse período situa-se entre 45 e 50 dias após a emergência (Pitelli \& Durigan, 1984; Pitelli, 1985).

A eficácia de controle das plantas daninhas com herbicidas residuais é influenciada pela umidade, pelos teores de argila e matéria orgânica, pelo pH e pela cobertura do solo com palha. Esses fatores influenciam processos de retenção, transferência e transformação destes herbicidas no solo, como adsorção, lixiviação e degradação biológica, e regulam a concentração e a persistência desses compostos no solo e, em função disso, a absorção pelas raízes de plantas daninhas (Moyer, 1987; Stougaard et al., 1990; Walker et al., 1992).

Cloroacetamidas são herbicidas residuais utilizados para controle de plantas daninhas em pré-emergência nas culturas de soja, milho, café, feijão e cana-de-açúcar. Neste grupo químico destacam-se os herbicidas acetochlor, alachlor e metolachlor. A atividade destes herbicidas é influenciada pelos teores de argila e matéria orgânica, pela umidade do solo e pela cobertura do solo com palha (Banks \& Robinson, 1986; Rodrigues \& Almeida, 1995). A eficácia de controle de plantas daninhas e o impacto ambiental proporcionado por esses herbicidas podem, também, variar em função da formulação utilizada. Formulações que possibilitem a liberação controlada das moléculas herbicidas no ambiente podem amenizar a volatilização e a lixiviação destes compostos no solo (Wauchope et al., 1990; Gish et al., 1994).
Em estudo sobre atividade biológica, adsorção, eficácia de controle das plantas daninhas, mobilidade e persistência no solo do herbicida alachlor, utilizado na formulação concentrada emulsionável e microencapsulada, Vasilakoglou \& Eleftherohorinos (1997) verificaram maior atividade biológica e lixiviação e menor adsorção ao solo quando foi usado na formulação concentrada emulsionável; entretanto, não observaram variações de persistência e eficácia de controle de plantas daninhas entre as formulações utilizadas. Associado a isso, ao compararem o efeito dessas formulações sobre o movimento, a toxicidade à cultura da soja e o controle de plantas daninhas com alachlor e metribuzin, Fleming et al. (1992) constataram que a formulação microencapsulada resultou em maior concentração destes herbicidas na superfície do solo, mas não verificaram variações de controle de plantas daninhas e de toxicidade à cultura da soja entre as formulações utilizadas.

Dependendo da cultura, a baixa seletividade apresentada pelos herbicidas cloroacetamidas torna necessário o uso de protetores com a finalidade de viabilizar o uso desses compostos no controle das plantas daninhas. O protetor CGA 43089 ( $\alpha$-(cianometoximino)-benzacetonitrila) preservou o sorgo da injúria causada por metolachlor, permitindo o uso deste herbicida no controle de plantas daninhas nesta cultura (Ellis et al., 1980), enquanto o composto N-1-aril-N-2dicloroacetil glicina foi eficaz na proteção de sementes de milho contra a injúria proporcionada pelos herbicidas acetochlor, alachlor e metolachlor (Repasi et al., 1996).

Este trabalho teve por objetivo avaliar a eficácia de controle de plantas daninhas pelos herbicidas cloroacetamidas aplicados em diferentes concentrações e formulações, em solo sob semeadura direta e preparo convencional.

\section{MATERIAL E MÉTODOS}

O experimento foi conduzido na Estação Experimental Agronômica da Universidade Federal do Rio Grande do Sul (EEA/UFRGS), localizada no município de Eldorado do Sul, região fisiográfica da Depressão Central do RS, no ano agrícola de 2000/2001. O solo utilizado é classificado, segundo o Sistema Brasileiro de Classificação de Solos (Empresa... - EMBRAPA, 
1999), como Argissolo Vermelho distrófico típico, contendo $28 \%$ de argila e manejado sob semeadura direta (sete anos consecutivos) e preparo convencional (aração e gradagens duas vezes ao ano).

Os herbicidas cloroacetamidas foram aplicados com pulverizador costal pressurizado com $\mathrm{CO}_{2}$, mantido à pressão constante de $200 \mathrm{kPa}$ e munido de bicos tipo leque 8002 , distanciados entre si em $0,50 \mathrm{~m}$ na barra de 1,5 m de largura, com volume de calda aspergida de $200 \mathrm{~L} \mathrm{ha}^{-1}$. Na semeadura direta, a cobertura do solo existente antes da aplicação dos tratamentos consistia de palha de aveia (Avena strigosa) na quantidade de 4,0 t ha ${ }^{-1} \mathrm{de}$ biomassa seca e a vegetação existente sobre a superfície do solo foi dessecada com o herbicida glyphosate a $1.080 \mathrm{~g}$ hă, no dia da implantação do experimento. As principais plantas daninhas presentes na área experimental eram Digitaria sp. (milhã), Bidens pilosa (picãopreto), Sida sp. (guanxuma) e Brachiaria plantaginea (papuã).

O delineamento experimental utilizado foi o de blocos casualizados com três repetições, sendo os tratamentos distribuídos em parcelas subdivididas. As unidades experimentais mediram $2 \times 6 \mathrm{~m}$. As parcelas foram constituídas pelos seguintes tratamentos: nas parcelas principais encontrava-se o preparo do solo (semeadura direta e preparo convencional); nas subparcelas, os herbicidas (acetochlor e alachlor na dose de $3.360 \mathrm{~g}$ hä; metolachlor, a $2.400 \mathrm{~g}$ ha; s-metolachlor, a $1.440 \mathrm{~g} \mathrm{had}$ s-metolachlor + protetor, a 1.440 e $1.800 \mathrm{~g}$ ha; e testemunha sem herbicida); e, nas subsubparcelas, as épocas de avaliação de controle das plantas daninhas (30 e 50 dias após a aplicação dos tratamentos - DAT). Além do controle, foram avaliadas matéria seca e população das plantas daninhas aos 30 e 50 DAT. O controle de plantas daninhas com os herbicidas acetochlor, alachlor e metolachlor foi avaliado visualmente, atribuindo-se notas que variaram de 0 para ausência a 100 para controle total destas plantas. A matéria seca e as populações de plantas daninhas foram avaliadas em área de $0,25 \mathrm{~m}^{2}$, coletadas aleatoriamente no interior de cada parcela. Após a coleta, as plantas foram secas em estufa a $60{ }^{\circ} \mathrm{C}$, até atingirem peso constante.
Na análise estatística, os dados relativos às porcentagens de controle de plantas daninhas sofreram transformação por arco-seno $\sqrt{\mathrm{x} / 100}$ ao serem submetidos à análise de variância. Os efeitos de preparo de solo, herbicidas e épocas de avaliação do controle de plantas daninhas foram avaliados mediante comparação das médias de tratamentos pelo teste de Tukey $(\mathrm{P} \leq 0,05)$.

\section{RESULTADOS E DISCUSSÃO}

O controle de plantas daninhas variou em função do sistema de preparo do solo, do tratamento herbicida e da época de avaliação (Tabela 1). Os herbicidas cloroacetamidas foram mais eficientes no controle de plantas daninhas no preparo convencional, comparado à semeadura direta, em ambas as épocas de avaliação. Os níveis de controle demonstram que estes herbicidas apresentam maior atividade biológica e persistência no preparo convencional.

Considerando-se o período total de prevenção da interferência e $80 \%$ como nível mínimo de controle necessário para prevenir reduções de produtividade das culturas devido à interferência causada pela presença de plantas daninhas nas lavouras (Pitelli \& Durigan, 1984; Pitelli, 1985; Fleck et al., 1989), os herbicidas acetochlor, alachlor, metolachlor e s-metolachlor + protetor, nas respectivas doses testadas, proporcionaram controle satisfatório das plantas daninhas somente no preparo convencional. Na semeadura direta, o controle destas plantas foi insuficiente em qualquer das épocas de avaliação.

Comparado aos demais herbicidas, o s-metolachlor + protetor a $1.800 \mathrm{~g}$ ha apresentou controle de plantas daninhas equivalente ao do acetochlor, alachlor e metolachlor nas doses de $3.360,3.360$ e $2.400 \mathrm{~g}$ hat, respectivamente. Ao comparar a atividade do metolachlor e seu isômero, o s-metolachlor, O' Connell (1998), citado por Grichar et al. (2001), também observou que s-metolachlor apresentou controle de plantas daninhas similar ao do metolachlor, porém com o uso de doses menores. No caso específico de Cyperus esculentus (tiririca), o s-metolachlor a $2.200 \mathrm{~g}$ hd apresentou controle similar ao do metolachlor utilizado a $4.500 \mathrm{~g}$ hà (Grichar al., 2001). Esse

Planta Daninha, Viçosa-MG, v.21, n.1, p.131-136, 2003 
resultado assume relevância ambiental, porque o uso do s-metolachlor permite alcançar a mesma eficácia de controle de plantas daninhas, porém com menor entrada de herbicida no ambiente.

De forma semelhante ao que foi observado no controle de plantas daninhas, o efeito dos tratamentos herbicidas sobre a produção de matéria seca também resultou em interação significativa de sistemas de preparo do solo, herbicidas e época de avaliação (Tabela 2). Em razão do controle mais eficiente, as plantas produziram menos matéria seca no preparo convencional em relação à semeadura direta, em ambas as épocas de avaliação. Esse resultado reforça a maior atividade biológica e persistência desses herbicidas no preparo convencional.

As populações das plantas daninhas variaram em função de épocas de avaliação e de

Tabela 1 - Valores médios de controle de plantas daninhas em função da aplicação de herbicidas cloroacetamidas (A), do preparo do solo (B) e das épocas de avaliação (C). EEA/UFRGS, Eldorado do Sul-RS, 2000/2001

\begin{tabular}{|c|c|c|c|c|c|}
\hline \multirow{3}{*}{ Herbicida } & \multirow{3}{*}{$\begin{array}{c}\text { Dose } \\
\left(\mathrm{g} \mathrm{ha}^{-1}\right)\end{array}$} & \multicolumn{2}{|c|}{ Semeadura direta } & \multicolumn{2}{|c|}{ Preparo convencional } \\
\hline & & $30 \mathrm{DAT}^{1 /}$ & $50 \mathrm{DAT}$ & $30 \mathrm{DAT}$ & $50 \mathrm{DAT}$ \\
\hline & & \multicolumn{4}{|c|}{$(\%)$} \\
\hline Acetochlor & 3.360 & ${ }^{2 /} \mathrm{S} 48 \mathrm{Aa}^{3 /}$ & $\mathrm{F} 12 \mathrm{Ab}$ & s $91 \mathrm{Aa}$ & f $86 \mathrm{Ab}$ \\
\hline Alachlor & 3.360 & $\mathrm{~S} 40 \mathrm{ABa}$ & $\mathrm{F} 10 \mathrm{Ab}$ & s $92 \mathrm{Aa}$ & f $85 \mathrm{Ab}$ \\
\hline Metolachlor & 2.400 & S $39 \mathrm{ABa}$ & $\mathrm{F} 11 \mathrm{Ab}$ & s $88 \mathrm{Aa}$ & f $79 \mathrm{Ab}$ \\
\hline s-Metolachlor & 1.440 & S $29 \mathrm{Ba}$ & $\mathrm{F} 7 \mathrm{Ab}$ & s $84 \mathrm{Aa}$ & f $64 \mathrm{Bb}$ \\
\hline s-Metolachlor+protetor & 1.440 & $\mathrm{~S} 32 \mathrm{Ba}$ & $\mathrm{F} 9 \mathrm{Ab}$ & s $88 \mathrm{Aa}$ & f $77 \mathrm{ABb}$ \\
\hline s-Metolachlor+protetor & 1.800 & $\mathrm{~S} 38 \mathrm{ABa}$ & $\mathrm{F} 16 \mathrm{Ab}$ & s $90 \mathrm{Aa}$ & f $86 \mathrm{Aa}$ \\
\hline Testemunha & --- & $\mathrm{S} 0 \mathrm{Ca}$ & $\mathrm{F} \quad 0 \mathrm{Ba}$ & $\mathrm{S} \quad 0 \mathrm{Ba}$ & $\mathrm{F} \quad 0 \mathrm{Ca}$ \\
\hline $\mathrm{CV}(\mathrm{A}) \%^{\underline{4}}$ & & \multicolumn{4}{|c|}{2,9} \\
\hline $\mathrm{CV}$ (B) $\%$ & & \multicolumn{4}{|c|}{9,6} \\
\hline $\mathrm{CV}(\mathrm{C}) \%$ & & \multicolumn{4}{|c|}{9,4} \\
\hline
\end{tabular}

${ }^{1 /}$ DAT $=$ dias após aplicação dos tratamentos. ${ }^{2 /}$ Médias antecedidas pela mesma letra maiúscula na linha não diferem pelo teste de Tukey $(\mathrm{P}>0,05)$. ${ }^{3} /$ Médias seguidas pela mesma letra maiúscula na coluna e minúscula na linha não diferem a $5 \%$ de probabilidade pelo teste de Tukey. ${ }^{4 /} \mathrm{CV}=$ coeficiente de variação.

Tabela 2 - Valores médios de biomassa de plantas daninhas em função da aplicação de herbicidas cloroacetamidas (A), do preparo do solo (B) e das épocas de avaliação (C). EEA/UFRGS, Eldorado do Sul-RS, 2000/2001

\begin{tabular}{|c|c|c|c|c|c|}
\hline \multirow{3}{*}{ Herbicida } & \multirow{3}{*}{$\begin{array}{c}\text { Dose } \\
\left(\mathrm{g} \mathrm{ha}^{-1}\right)\end{array}$} & \multicolumn{2}{|c|}{ Semeadura direta } & \multicolumn{2}{|c|}{ Preparo convencional } \\
\hline & & $30 \mathrm{DAT}^{1 /}$ & $50 \mathrm{DAT}$ & $30 \mathrm{DAT}$ & $50 \mathrm{DAT}$ \\
\hline & & \multicolumn{4}{|c|}{$\left(\mathrm{g} \mathrm{m}^{-2}\right)$} \\
\hline Acetochlor & 3.360 & $\underline{2}^{2} \mathrm{~S} 6,8 \mathrm{Ab}^{\underline{3} /}$ & F $128 \mathrm{Aa}$ & $\mathrm{s} 1,0 \mathrm{Bb}$ & $\mathrm{f} 2,2 \mathrm{Ca}$ \\
\hline Alachlor & 3.360 & $\mathrm{~S} \mathrm{5,9} \mathrm{Ab}$ & F $126 \mathrm{Aa}$ & $\mathrm{s} 1,3 \mathrm{Bb}$ & f $5,0 \mathrm{BCa}$ \\
\hline Metolachlor & 2.400 & $\mathrm{~S} 6,1 \mathrm{Ab}$ & F $154 \mathrm{Aa}$ & $\mathrm{s} 1,4 \mathrm{Bb}$ & f $4,6 \mathrm{BCa}$ \\
\hline s-Metolachlor & 1.440 & $\mathrm{~S} 8,1 \mathrm{Ab}$ & F $128 \mathrm{Aa}$ & $\mathrm{s} 2,5 \mathrm{ABb}$ & $\mathrm{f} 9,9 \mathrm{Ba}$ \\
\hline s-Metolachlor+protetor & 1.440 & $\mathrm{~S} 10 \mathrm{Ab}$ & F $164 \mathrm{Aa}$ & $\mathrm{s} 3,2 \mathrm{ABb}$ & f $6,3 \mathrm{BCa}$ \\
\hline s-Metolachlor+protetor & 1.800 & $\mathrm{~S} 6,3 \mathrm{Ab}$ & F $161 \mathrm{Aa}$ & $\mathrm{s} 1,1 \mathrm{Bb}$ & $\mathrm{f} 3,3 \mathrm{Ca}$ \\
\hline Testemunha & --- & $\mathrm{S} 9,8 \mathrm{Ab}$ & F $165 \mathrm{Aa}$ & $\mathrm{S} 8,3 \mathrm{Ab}$ & f $25 \mathrm{Aa}$ \\
\hline $\mathrm{CV}(\mathrm{A}) \%^{\underline{4}}$ & & \multicolumn{4}{|c|}{3,2} \\
\hline CV (B) $\%$ & & \multicolumn{4}{|c|}{25,1} \\
\hline $\mathrm{CV}(\mathrm{C}) \%$ & & \multicolumn{4}{|c|}{45,9} \\
\hline
\end{tabular}

${ }^{1 /}$ DAT = dias após aplicação dos tratamentos. ${ }^{2} /$ Médias antecedidas pela mesma letra maiúscula na linha não diferem pelo teste de Tukey $(\mathrm{P}>0,05) .{ }^{3 /}$ Médias seguidas pela mesma letra maiúscula na coluna e minúscula na linha não diferem a $5 \%$ de probabilidade pelo teste de Tukey. ${ }^{4 /} \mathrm{CV}=$ coeficiente de variação. 
sistemas de preparo do solo (Tabela 3), bem como entre sistemas de preparo do solo e herbicidas (Tabela 4). Menor população de plantas daninhas foi observada no preparo convencional, demonstrando que os herbicidas cloroacetamidas apresentam-se mais ativos neste sistema de preparo (Tabela 3). A menor população de plantas daninhas observada no preparo convencional decorre da maior eficácia de controle destas plantas observada nesta forma de preparo do solo (Tabela $1)$.

A população de plantas variou de forma dife-

Tabela 3 - Valores médios de população de plantas daninhas em função do preparo do solo (A) e das épocas de avaliação (B). EEA/UFRGS, Eldorado do Sul-RS, 2000/ 2001

\begin{tabular}{|c|c|c|}
\hline \multirow{3}{*}{ Preparo do solo } & \multicolumn{2}{|c|}{ Época de avaliação } \\
\hline & $30 \mathrm{DAT}^{1}$ & $50 \mathrm{DAT}$ \\
\hline & \multicolumn{2}{|c|}{ (plântulas $\mathrm{m}^{-2}$ ) } \\
\hline Semeadura direta & $66 \mathrm{Aa}^{2 /}$ & $54 \mathrm{Ab}$ \\
\hline Preparo convencional & $7 \mathrm{Bb}$ & $14 \mathrm{Ba}$ \\
\hline $\mathrm{CV}(\mathrm{A}) \%^{\frac{3}{}}$ & \multicolumn{2}{|c|}{22,5} \\
\hline $\mathrm{CV}(\mathrm{B}) \%$ & \multicolumn{2}{|c|}{23,9} \\
\hline
\end{tabular}

${ }^{1 /}$ DAT $=$ dias após aplicação dos tratamentos. ${ }^{2 /}$ Médias seguidas pela mesma letra maiúscula na coluna e minúscula na linha não diferem a $5 \%$ de probabilidade pelo teste de Tukey. ${ }^{3 /} \mathrm{CV}=$ coeficiente de variação.

renciada no tempo entre os sistemas de preparo do solo. Na semeadura direta, a população de plantas reduziu-se no período entre 30 e 50 DAT, enquanto no preparo convencional ocorreu o inverso (Tabela 3). O incremento da competição intra-específica, decorrente da maior população de plantas daninhas na semeadura direta (Tabela 3), pode ter condicionado esse resultado.

A população de plantas daninhas variou para os herbicidas entre as formas de preparo do solo (Tabela 4), sendo menor no preparo convencional. A maior eficácia de controle e a maior persistência apresentadas por esses herbicidas no preparo convencional (Tabela 1) conduziu a esse resultado. A atividade e a persistência dos herbicidas cloroacetamidas podem variar em função da lixiviação, da adsorção ao solo e à palha e da degradação e/ou transformação biológica (Beestman \& Deming, 1974; Peter \& Weber, 1985;
Banks \& Robinson, 1986; Pothuluri et al., 1990), sendo estas possíveis explicações para a variação de controle das plantas daninhas observada entre a semeadura direta e o preparo convencional. A palha, sobretudo, afeta a atividade de herbicidas cloroacetamidas. Menos de $10 \%$ do herbicida atingiu o solo na presença de palha na quantidade de $4.480 \mathrm{~kg} \mathrm{ha}{ }^{1}$, sugerindo que a eficácia de controle das plantas daninhas pode ser reduzida com sua presença, segundo Banks \& Robinson, (1986). Além disso, Burgos \& Talbert (1996) observaram que o herbicida metolachlor foi mais eficaz no controle destas plantas na ausência da cobertura do solo do que na presença. Dessa forma, o menor controle das plantas daninhas apresentado pelos herbicidas cloroacetamidas na semeadura direta pode ser decorrente da adsorção desses compostos à palha.

Conclui-se que os herbicidas cloroacetamidas apresentaram maior eficácia de controle de plantas daninhas em Argissolo Vermelho sob

Tabela 4 - Valores médios de população de plantas daninhas em função da aplicação de herbicidas cloroacetamidas (A) e do preparo do solo (B). EEA/UFRGS, Eldorado do Sul-RS, 2000/2001

\begin{tabular}{|l|c|c|c|}
\hline \multirow{2}{*}{ Herbicida } & \multirow{2}{*}{$\begin{array}{c}\text { Dose } \\
\left(\mathrm{g} \mathrm{ha}^{-1}\right)\end{array}$} & $\begin{array}{c}\text { Semeadura } \\
\text { direta }\end{array}$ & $\begin{array}{c}\text { Preparo } \\
\text { convencional }\end{array}$ \\
\cline { 3 - 4 } & & \multicolumn{2}{|c|}{ (plântulas m$^{-2}$ ) } \\
\hline Acetochlor & 3.360 & $40 \mathrm{Aa}^{1 /}$ & $6 \mathrm{ABb}$ \\
Alachlor & 3.360 & $44 \mathrm{Aa}$ & $7 \mathrm{ABb}$ \\
Metolachlor & 2.400 & $56 \mathrm{Aa}$ & $4 \mathrm{Ab}$ \\
s-Metolachlor & 1.440 & $96 \mathrm{Aa}$ & $10 \mathrm{ABb}$ \\
s-Metolachlor+protetor & 1.440 & $60 \mathrm{Aa}$ & $5 \mathrm{ABb}$ \\
s-Metolachlor+protetor & 1.800 & $62 \mathrm{Aa}$ & $4 \mathrm{Ab}$ \\
Testemunha & ---- & $61 \mathrm{Aa}$ & $35 \mathrm{Bb}$ \\
\hline CV (A) \% ${ }^{2 /}$ & & \multicolumn{2}{|c}{22,5} \\
CV (B) \% & & \multicolumn{2}{|c}{47,1} \\
\hline
\end{tabular}

1/ Médias seguidas pela mesma letra maiúscula na coluna e minúscula na linha não diferem a $5 \%$ de probabilidade pelo teste de Tukey $(\mathrm{P}>0,05) .{ }^{2 /} \mathrm{CV}=$ coeficiente de variação.

preparo convencional, comparado à semeadura direta.

\section{LITERATURA CITADA}

Planta Daninha, Viçosa-MG, v.21, n.1, p.131-136, 2003 
BANKS, A. P.; ROBINSON, E. L. Soil reception and activity of acetochlor, alachlor, and metolachlor as affected by wheat (Triticum aestivum) straw and irrigation. Weed Sci., v. 34, n. 3, p. 607-611, 1986.

BEESTMAN, G. B.; DEMING, J. M. Dissipation of acetanilide herbicides from soils. Agron. J., v. 66, n. 1, p. 308-311, 1974.

BURGOS, N. R.; TALBERT, R. E. Weed control and sweet corn (Zea mays var. rugosa) response in a no-till system with cover crops. Weed Sci., v. 44, n. 2, p. 355-361, 1996.

DURIGAN, J. C. et al. Períodos de matocompetição na cultura da soja (Glycine max (L.) Merrill), cultivares Santa Rosa e IAC-2. I. Efeitos sobre os parâmetros de produção. Planta Daninha, v. 2, n. 1, p. 86-100, 1983.

ELLIS, J. F. et al. Effectiveness of a new safener for protecting sorghum (Sorghum bicolor) from metolachlor injury. Weed Sci., v. 28, n. 1, p. 1-5, 1980.

EMPRESA BRASILEIRA DE PESQUISA AGROPECUÁRIA-EMBRAPA. Serviço Nacional de Pesquisa de Solos. Sistema Brasileiro de Classificação de Solo. Rio de Janeiro, 1999. 412 p.

FLECK, N. G.; MENGARDA, I. P.; PINTO, J. J. O. Interferência de plantas daninhas na cultura de girassol. Competição no tempo. Pesq. Agropec. Bras., v. 24, n. 9 , p. 1139-1147, 1989.

FLEMING, G. F. et al. Movement of alachlor and metribuzin from controlled release formulations in a sandy soil. Weed Sci., v. 40, n. 2, p. 606-613, 1992.

GISH, T. G.; SHIRMOHAMMADI, A.; WIENHOLD, B. J. Field-scale mobility and persistence of commercial and starch-encapsulated atrazine and alachlor. J. Environ. Quality, v. 23, n. 1, p. 355-359, 1994.

GRICHAR, W. J. et al. S-metolachlor compared with metolachlor on yellow nutsedge (Cyperus esculentus) and peanut (Arachis hypogaea). Weed Technol., v. 15, n. 1, p. 107-111, 2001.

MOYER, J. R. Effect of soil moisture on the efficacy and selectivity of soil-applied herbicides. Rev. Weed Sci., v. 3, p. 19-34, 1987.
PETER, C. J.; WEBER, B. J. Adsorption, mobility and efficacy of alachlor and acetochlor as influenced by soil properties. Weed Sci., v. 33, n. 3, p. 874-881, 1985.

PITELLI, R. A. Interferência de plantas daninhas em culturas agrícolas. Inf. Agropec., v. 11, n. 129, p. 16-27, 1985.

PITELLI, R. A.; DURIGAN, J. C. Terminologia para períodos de controle e de convivência das plantas em culturas anuais e bianuais. In: CONGRESSO BRASILEIRO DE HERBICIDAS E PLANTAS DANINHAS, 15., 1984, Belo Horizonte. Resumos... Belo Horizonte: SBHED, 1984. p. 34

POTHULURI, J. V. et al. Aerobic and anaerobic degradation in samples from a surface-to-groundwater profile. J. Environ. Quality, v. 19, n. 2, p. 525-530, 1990.

REPASI, J. et al. N-1-aryl-N2-dichoroacetyl glycine and alanineamides as selective safeners for corn against chloroacetanilide herbicides. J. Environ. Sci. Health, v. 31, p. $567-571,1996$.

RODRIGUES, B. N.; ALMEIDA, F. S. Guia de herbicidas. 3.ed. Londrina: 1995. $675 \mathrm{p}$.

STOUGAARD, R. N.; SHEA, P. J.; MARTIN, A. R. Effect of soil type and $\mathrm{pH}$ on adsorption, mobility and efficacy of imazaquin and imazethapyr. Weed Sci., v. 38, n. 1, p. 6773,1990 .

VASILAKOGLOU, I. B.; ELEFTHEROHORINOS, I. G. Activity, adsorption, mobility, efficacy and persistence of alachlor as influenced by formulation. Weed Sci., v. 45, n. 2, p. 579-585, 1997.

WALKER, A.; MOON, Y.; WELCH., S. J. Influence of temperature, soil moisture and soil characteristics on the persistence of alachlor. Pest. Sci., v. 35, n. 1, p. 109-116, 1992.

WAUCHOPE, R. D.; WILLIAMS, R. G.; MARTI, L. R. Runoff of sulfometurom-methil and cyanazine from small plots: effects of formulation and grass cover. J. Environ. Quality, v. 19, n. 1, p. 119-125, 1990. 